\title{
Covalently Attached Antimicrobial Surfaces Using BODIPY: Improving Efficiency and Effectiveness
}

William J. Peveler, ${ }^{*}, \dagger \bullet$ Sacha Noimark, ${ }^{\dagger, \dagger}$ Hassan Al-Azawi, ${ }^{\dagger}$ Gi Byoung Hwang, ${ }^{\dagger}$ Colin R. Crick, ${ }^{\| \bullet}$ Elaine Allan, ${ }^{\S}$ Joshua B. Edel, ${ }^{\perp}{ }^{\circledR}$ Aleksandar P. Ivanov, ${ }^{\perp}$ Alexander J. MacRobert, ${ }^{\sharp}$ and Ivan P. Parkin ${ }^{*}$,

${ }^{\dagger}$ Department of Chemistry, University College London, 20 Gordon Street, London WC1H 0AJ, U.K.

${ }^{\ddagger}$ Department of Medical Physics and Biomedical Engineering, Malet Place Engineering Building, University College London, London WC1E 6BT, U.K.

${ }^{\S}$ Division of Microbial Diseases, UCL Eastman Dental Institute, University College London, 256 Gray’s Inn Road, London WC1X 8LD, U.K.

${ }$ Materials Innovation Factory, Department of Chemistry, University of Liverpool, Liverpool L69 7ZD, U.K.

${ }^{\perp}$ Department of Chemistry, Imperial College London, Exhibition Road, London SW7 2AZ, U.K.

\# Division of Surgery \& Interventional Science, Department of Nanotechnology, Royal Free Campus, University College London, Rowland Hill Street, London NW3 2PF, U.K.

\section{Supporting Information}

ABSTRACT: The development of photoactivated antimicrobial surfaces that kill pathogens through the production of singlet oxygen has proved very effective in recent years, with applications in medical devices and hospital touch surfaces, to improve patient safety and well being. However, many of these surfaces require a swell-encapsulation-shrink strategy to incorporate the photoactive agents in a polymer matrix, and this is resource intensive, given that only the surface fraction of the agent is active against bacteria. Furthermore, there is a risk that the agent will leach from the polymer and thus raises issues of biocompatibility and patient safety. Here, we describe a more efficient method of fabricating a silicone material with a covalently attached monolayer of photoactivating agent that uses heavy-atom triplet sensitization for improved singlet oxygen generation and corresponding antimicrobial activity. We use borondipyrromethane with a reactive end group and incorporated $\mathrm{Br}$ atoms, covalently attached to poly(dimethylsiloxane). We demonstrate the efficacy of this material in producing singlet oxygen and killing Staphylococcus aureus and suggest how it might be easily modifiable for future antimicrobial surface development.

KEYWORDS: BODIPY, antimicrobial, PDMS, singlet oxygen, fluorescence microscopy

\section{INTRODUCTION}

Healthcare-associated infections (HAIs) are a significant financial burden on healthcare institutions, and can result in extended patient hospitalization durations, patient discomfort, and mortality. ${ }^{1}$ These infections are associated with medical interventions (e.g., catheterization) or can be due to contact with contaminated surfaces in hospitals. ${ }^{2}$

One approach to reduce the incidence of infection is the use of self-sterilizing surfaces to help decrease the risk of infection upon use of medical devices such as catheters and also to disrupt the cycle of transmission of bacteria on touch surfaces in hospitals. ${ }^{3}$ Potential methods tested include using inherently antimicrobial materials such as copper, or surfaces bearing pendant quaternary ammonium ions, as well as altering the hydrophobicity/philicity of the surface. ${ }^{4,5}$ One effective strategy demonstrated recently is the use of photodynamic therapy to reduce bacterial loads on heavily contaminated surfaces. ${ }^{6}$

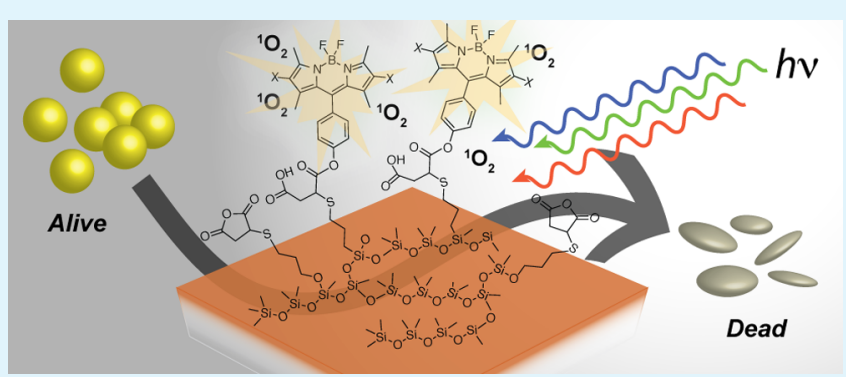

To create photodynamic surfaces, suitable dyes can be readily incorporated into medical grade polymers, using a simple swellencapsulation-shrink strategy, with the aim of generating reactive oxygen species (ROS) such as singlet oxygen, when illuminated with white light. ${ }^{7}$ These ROS initiate a nonsite specific attack against bacteria, killing them without inducing resistance. ${ }^{8,9}$ However, limitations to the swell-encapsulationshrink strategy include high polymer coloration, in addition to a nonoptimal generation of key ROS at the polymer surface (within the vicinity of bacteria), as described below. ${ }^{10}$

The existing work on this type of antibacterial surface has focused on the use of swell-encapsulation-shrink methodology with phenothiazine dyes, such as toluidine blue or methylene

Received: September 1, 2017

Accepted: December 6, 2017

Published: December 6, 2017 


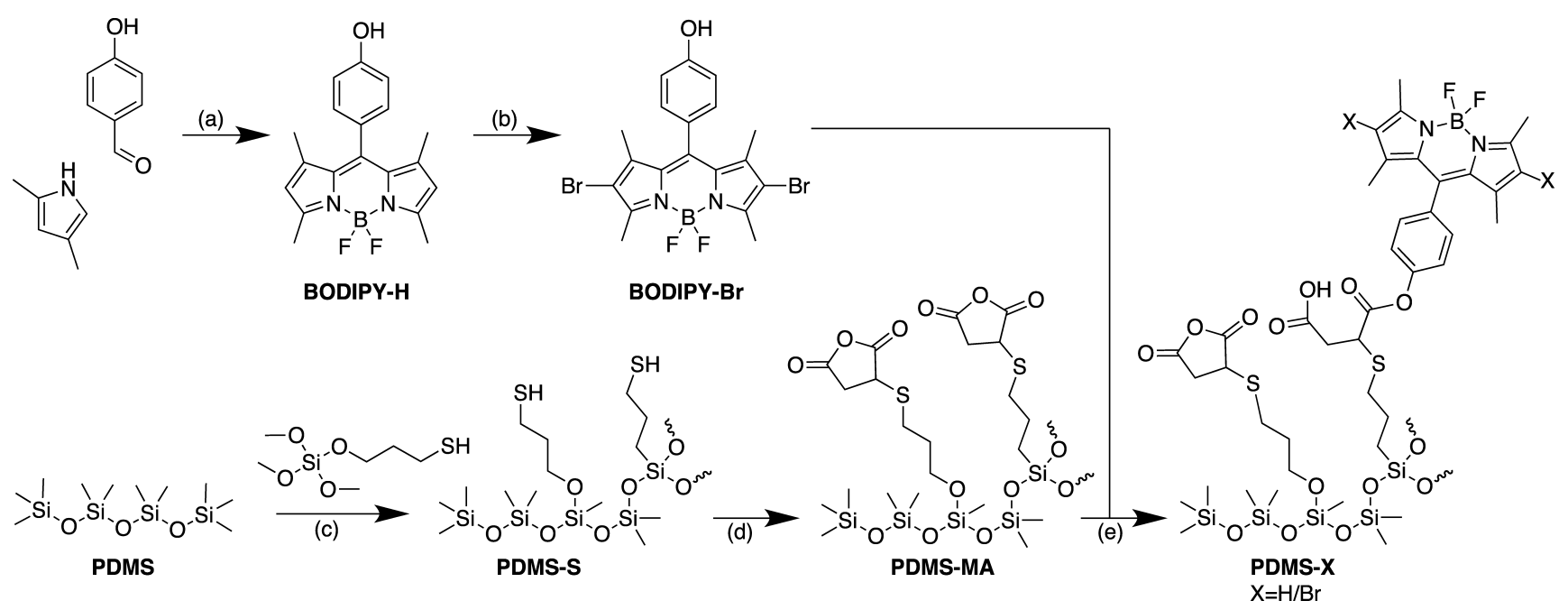

Figure 1. Scheme of BODIPY synthesis and PDMS modification. (a) Trifluoroacetic acid, tetrahydrofuran (THF), $18 \mathrm{~h}$; then, 2,3-dichloro-5,6dicyano-1,4-benzoquinone (DDQ), 4 h; then, triethylamine, $\mathrm{BF}_{3} \cdot \mathrm{OEt}_{2}, 18 \mathrm{~h}, 44 \%$; (b) $\mathrm{Br}_{2}$ dropwise, $\mathrm{CH}_{2} \mathrm{Cl}_{2}, 3 \mathrm{~h}, 69 \%$; (c) $\mathrm{KOH}, \mathrm{MeOH}, 8 \mathrm{~h}, 50$ ${ }^{\circ} \mathrm{C}$; (d) maleic anhydride, THF, AIBN, $48 \mathrm{~h}, 80{ }^{\circ} \mathrm{C}$; (e) $\mathrm{CH}_{2} \mathrm{Cl}_{2}, 72 \mathrm{~h}$.

blue. ${ }^{7,11}$ However, these materials do not inherently generate a large amount of ROS on irradiation and so require the presence of gold nanoparticles to enhance triplet generation via intersystem crossing (ISC), leading to type II photosenitization and reactive triplet species that react with atmospheric oxygen to generate bactericidal singlet oxygen $\left({ }^{1} \mathrm{O}_{2}\right) \cdot{ }^{12}$ This relies upon using expensive materials such as gold to ensure efficacious bacterial kills. ${ }^{13}$ Furthermore, swell-encapsulated materials could be susceptible to leaching of the photosensitizer or nanomaterials by washing, or by repeated contact, presenting an environmental hazard. Other cheaper nanomaterials have been explored with success, such as $\mathrm{ZnO}$ and $\mathrm{Cu}$ particles, ${ }^{6,14,15}$ but issues associated with the two-component system remain, along with the potential cytotoxicity of such nanomaterials. Finally, there is the issue of material wastage by trapping it in the bulk of the polymer, away from the surface, where it cannot impact the microorganisms due to the short path length of any ROS generated within the polymeric matrix. ${ }^{16,17}$

The covalent attachment of the most effective triarylmethane dyes (crystal violet) to the nanoparticle or polymeric material may surmount many of the aforementioned problems; however, synthetically, it is nontrivial and in cases where colocation with nanomaterials is desirable, it becomes difficult to ensure the nanoparticles are located at or near the surface. ${ }^{18,19}$ Here, we have searched for known efficient triplet producing dyes that have a modular (easily modifiable) structure to allow for ease of covalent attachment and good ROS generation without nanomaterial incorporation. Some previous work has focused on porphyrins; ${ }^{18}$ however, despite having the modularity, synthesis can be challenging and precious metal salts are required for the porphyrin core to induce good ROS activity. ${ }^{19}$

One promising although less explored candidate is borondipyrromethane (BODIPY). BODIPY has been shown to be an effective generator of ROS and therefore useful for this class of light-activated antimicrobial surfaces. ${ }^{20,21}$ It is also noncytotoxic to mammalian cells in its own right (although can be modified to be inherently bactericidal without the application of light via addition of quaternary ammonium cations $)^{22}$ and has easily tailorable chemistry for the inclusion of synthetic handles to covalently couple it to other materials. ${ }^{23}$
The linking of dyes to substrates is an effective and widely applicable technique that has been shown to work on cellulose paper, ${ }^{23}$ and the synthetic strategy can be modified to extend this surface attachment technology to other polymers commonly used in healthcare environments such as polyurethane. $^{18,24}$ This will be aided by the modularity of the BODIPY ROS generator, giving several potential chemical attachment routes.

Herein, we have synthesized a triplet generating BODIPY species that produces singlet oxygen species for antimicrobial effect. We subsequently covalently coupled it to the surface of a sample polymer, poly(dimethylsiloxane) (PDMS), widely used in medical devices, for the first time. Covalent coupling is demonstrated through Raman, IR, and fluorescence microscopy, and the material is shown to produce good singlet oxygen yields at the surface. This surface attachment and subsequent reclamation/recycling of unattached BODIPY reduces the wastage of material in the polymer bulk. Finally, we demonstrate effective light-activated antibacterial activity against Staphylococcus aureus and Escherichia coli, pathogens commonly associated with HAIs.

\section{RESULTS AND DISCUSSION}

Synthesis, Characterization, and Singlet Oxygen Testing. Thin (ca. $1 \mathrm{~mm}$ ) PDMS substrates were prepared as described in the Methods section and then thiolated with (3mercaptopropyl)trimethoxysilane (MTS) using the method of Brook et al., ${ }^{25}$ with $\mathrm{KOH}$ as the base. These substrates were washed thoroughly, and thiolation was confirmed by presence of a characteristic $\mathrm{S}-\mathrm{H}$ stretch at $2583 \mathrm{~cm}^{-1}$ in the Raman spectrum (Figure S1). Maleic anhydride (MA) was then attached with thiolene click chemistry (initiated with azobisisobutyronitrile (AIBN)) to give a polymer surface to which nucleophiles could be attached (Figure 1). The complete attachment of MA could be inferred from loss of the $\mathrm{S}-\mathrm{H}$ stretch in the Raman spectrum and the presence of new carbonyl stretches in the IR spectrum (Figures S1 and S2).

A BODIPY with open $(-\mathrm{H})$ 2,6 positions was synthesized with a meso-phenolic group, which could act as an attachment point to the anhydride. This material was then halogenated with bromine at the 2,6 positions to improve intersystem 
(a)

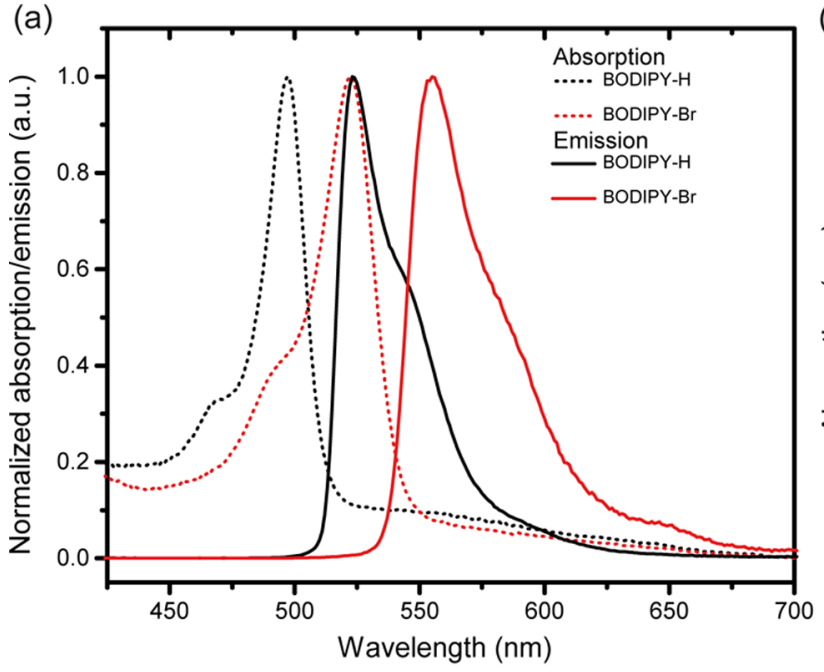

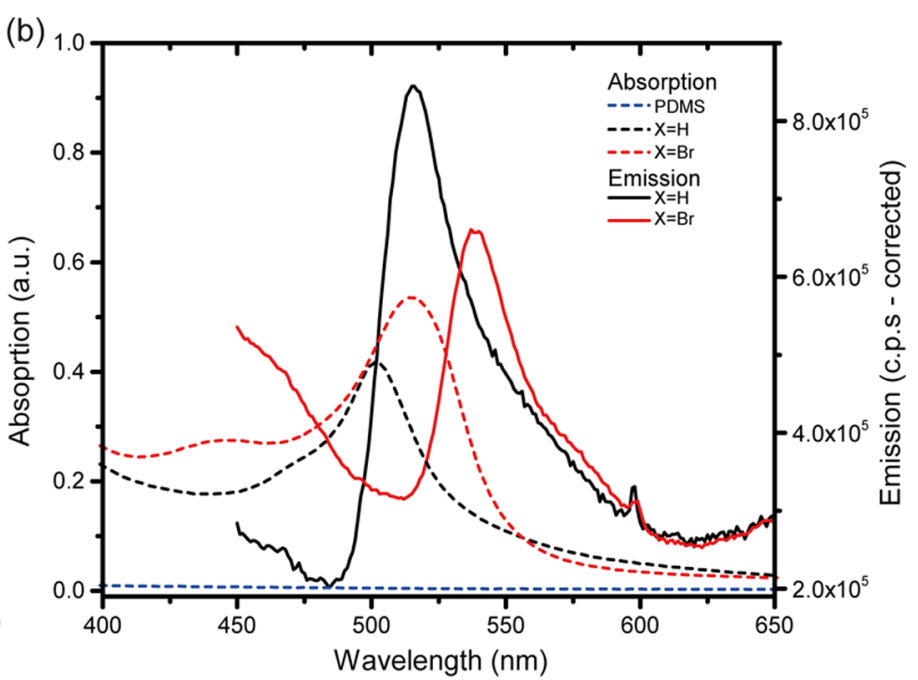

Figure 2. Photocharacterization of BODIPY in $\mathrm{MeOH}$ solution (normalized (a)) and on PDMS (b).

crossing (ISC) for triplet generation (Figure 1). The bromination of the BODIPY at the 2,6 positions redshifts the absorption and fluorescence by 25 and $32 \mathrm{~nm}$, respectively, decreases the photoluminescence (PL) quantum yield from 36.4 to $7.6 \%$, and decreases principal fluorescence lifetime from 3.96 to 1.43 ns due to the improved ISC (Figure 2a, Table 1).

Table 1. Summary of Singlet Oxygen Lifetime Measurements for Solution and Polymeric Materials ${ }^{a}$

$\begin{array}{llcll}\text { material } & & \tau_{1} & \tau_{2} & \text { relative }{ }^{1} \mathrm{O}_{2} \mathrm{QY} \\ \text { solution } & \text { BODIPY-H } & 33.61 & 0.005 & \leq 0.05(\leq 0.01) \\ & \text { BODIPY-Br } & 33.35 & 0.26 & 1.00(0.17) \\ & \text { RB } & 33.77 & 0.44 & 4.77(0.79) \\ \text { PDMS } & \text { PDMS-H } & 36.73 & 0.98 & 0.24 \\ & \text { PDMS-Br } & 45.27 & 1.36 & 1.00\end{array}$

${ }^{a} \tau$ is measured in $\mu$ s. Solution quantum yield (QY) measured relative to Rose Bengal ( $\mathrm{RB}$ ) standard is given in brackets for solution samples.

The BODIPY was attached to the MA-PDMS by facile nucleophilic addition to the MA moieties, and then the polymer was soaked in $\mathrm{CH}_{2} \mathrm{Cl}_{2}$ to remove any swell-encapsulated material (evidenced visually and by IR, Figure S2 and microscopy below). This BODIPY solution could be recovered and used repeatedly to surface-functionalize multiple polymer samples. Scanning electron microscopy imaging of the PDMS (Figure S3) showed that there were some surface morphology changes to the polymer ${ }^{26}$ but the basic mechanical integrity was maintained, despite the vigorous washing regimes. This is attributed to the fact that only the surface of the PDMS is chemically modified, so the bulk material retains its properties.

The raw PDMS for the substrate showed no strong absorption or emission in the visible region; however, on thiolation and addition of MA, a long-lived fluorescence $(\sim 8$ ns) was also observed at ca. $450 \mathrm{~nm}$ and is suggested to originate from the thiolation of PDMS (see Table S1). The final PDMS-BODIPY showed absorption and fluorescence characteristics similar to that of the unattached dye, but with a slight blue shift and broadening of the bands, due to the solidstate nature of the sample and different microenvironment $\left(\lambda_{\mathrm{em}}(\mathrm{H})=525 \mathrm{~nm}, \lambda_{\mathrm{em}}(\mathrm{Br})=553 \mathrm{~nm}\right.$, compared in Figure S4). The samples also exhibited increased fluorescence lifetimes for the principal emission (4.35 and $2.52 \mathrm{~ns}$, respectively), likely due to a reduction in free rotation of the BODIPY moiety within the polymer matrix. Additionally, there is an apparent increase in the number of alternative decay pathways (an increase in the number of fluorescent lifetimes in the signal) due to fluorescence relaxation through the polymer matrix as well as other nonradiative mechanisms. ${ }^{10}$

Fluorescence imaging and lifetime imaging (Figures 3 and S5) were used to investigate the dispersion of the BODIPY in cross sections of the PDMS. These images show that the washing regime removed all of the dye encapsulated within the polymer bulk, resulting in a thin layer of surface-bound BODIPY (ca. 50-100 $\mu \mathrm{m}$ ), indicating the covalent attachment regime was successful. A confocal z-stack was created to image the fluorescence and the lifetimes of the fluorophores in the PDMS samples. This approach is a more accurate method than previously used scalpel or microtome cutting of the PDMS for cross-sectional imaging, which can lead to scattering due to surface roughness or "smearing" of the dye segment (Figure S6). PL properties measured in this modality were similar to those originating from the previous bulk measurements, and the dye was shown to have been attached to a depth of ca. 50 $\mu \mathrm{m}$ into the PDMS. This is probably due to surface rearrangement, where the hydrophobic dye molecules reorient from the surface of the PDMS slightly into the bulk, enabled by the relatively flexible MA linker. Figure 3a,b shows threedimensional intensity and lifetime maps of a cross-sectional profile along the $90^{\circ}$ corner edge of the rectangular PDMS-Br sample. The samples showed both the highest surface concentration at the bottom surface and the outer edges of the PDMS-H and -Br samples, and a rough calculation based on estimated absorption coefficients for the dyes indicated a concentration of $5 \times 10^{4}$ BODIPY units $/ \mu \mathrm{m}^{2}$. No appreciable fluorescence was observed in the unmodified PDMS controls. Importantly, across samples, the lifetime remained the same, with components $\tau 1=0.4 \pm 0.2 \mathrm{~ns}$ and $\tau 2=2.2 \pm 0.3 \mathrm{~ns}$ for BODIPY-Br and $\tau 1=0.4 \pm 0.2 \mathrm{~ns}$ and $\tau 2=3.4 \pm 0.4 \mathrm{~ns}$ for BODIPY-H, respectively (Figures $3 \mathrm{e}$ and $\mathrm{S} 5 \mathrm{e}$ ). In both cases, the first lifetime component was associated with the instrument response function, whereas the second was slightly lower but comparable to lifetime values observed in bulk measurements (Table S1). Although the lifetime values remained very similar with increasing slice depth, the fluorescent intensity decreased exponentially, which indicated a substantial decrease of the 


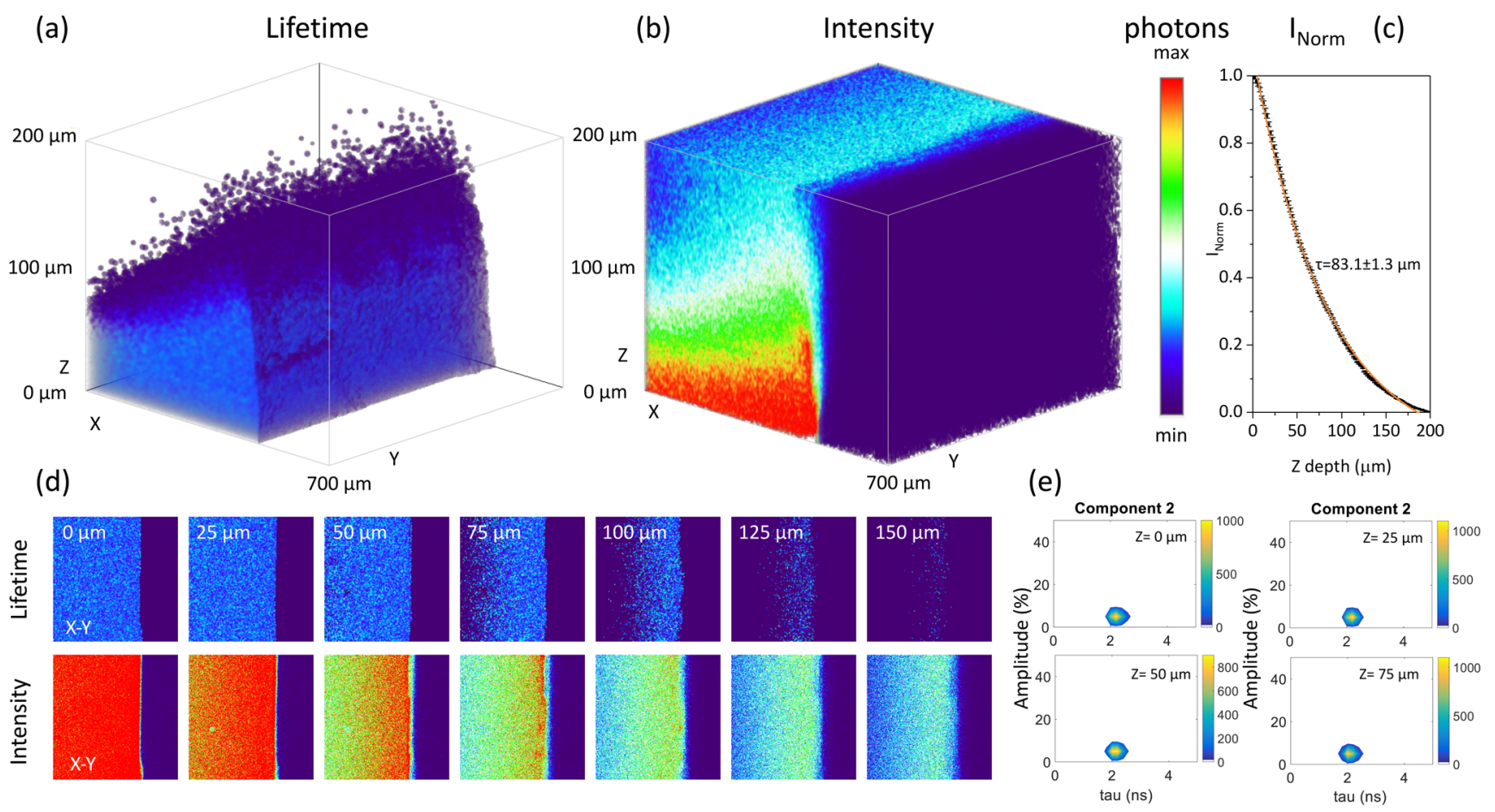

Figure 3. Fluorescence microscopy on PDMS-Br cross sections constructed from sequential z-scanning. (a) Lifetime and (b) intensity $3 \mathrm{D}$ maps (X$Y-Z 700 \mu \mathrm{m} \times 700 \mu \mathrm{m} \times 200 \mu \mathrm{m}$ ). Confocal slices were taken with $2 \mu \mathrm{m}$ step and averaged over 20 frames for each slice. (c) Normalized intensity over full slice depth $(0-200 \mu \mathrm{m})$, (d) 2D intensity and lifetime maps showing polymer $X Y$ cross section at $0-150 \mu \mathrm{m}$ slice depth (e) Lifetime scatter plots for second lifetime component at different slice depths. An equivalent plot for PDMS-H is given in Figure S4.

bound dye. We observed $1 / e$ decrease of intensity at $z=83.1 \pm$ $1.3 \mu \mathrm{m}$ for BODIPY-Br-functionalized samples and $z=48.3 \pm$ $0.6 \mu \mathrm{m}$ for BODIPY-H-functionalized samples in general agreement with the Rhodamine-functionalized materials produced by Brook et al. ${ }^{25}$

ROS Generation and Antibacterial Testing. Singlet oxygen $\left({ }^{1} \mathrm{O}_{2}\right)$ generation measurements were performed on airequilibrated solid phase and solution phase (MeOD) BODIPY samples using $532 \mathrm{~nm}$ laser excitation and phosphorescence detection of ${ }^{1} \mathrm{O}_{2}$ at $1270 \mathrm{~nm}$. The time-resolved near-IR ${ }^{1} \mathrm{O}_{2}$ response can be used to determine information on both the kinetics and quantity of ${ }^{1} \mathrm{O}_{2}$ production. The ${ }^{1} \mathrm{O}_{2}$ signal can be fitted with two components, lifetimes $\tau_{1}$ and $\tau_{2}$, providing information on the ${ }^{1} \mathrm{O}_{2}$ decay via collisional quenching with other species and the formation of ${ }^{1} \mathrm{O}_{2}$ from the dye triplet state, respectively. The intensity of the ${ }^{1} \mathrm{O}_{2}$ signal can also be used to calculate the relative ${ }^{1} \mathrm{O}_{2}$ quantum yields. ${ }^{10}$ For the solution measurements, Rose Bengal (RB) was used as a standard (with an absolute ${ }^{1} \mathrm{O}_{2}$ yield of 0.79). ${ }^{27}$ Both measurements are summarized in Table 1.

Table 1 shows that addition of $\mathrm{Br}$ to the BODIPY greatly improves its ${ }^{1} \mathrm{O}_{2}$ yield $\left({ }^{1} \mathrm{O}_{2} \mathrm{QY}_{\text {BODIPY-Br }} \gg{ }^{1} \mathrm{O}_{2} \mathrm{QY}_{\text {BODIPY-H }}\right)$ through a heavy-atom effect. ${ }^{28,29}$ The reduction in the PL quantum yield and fluorescence lifetimes leads to improved ISC, achieving increased triplet yields and concurrent singlet oxygen generation via a type II mechanism. ${ }^{20}$ This trend is maintained on attachment of the BODIPY to the PDMS, but there is also an increase in ${ }^{1} \mathrm{O}_{2}$ yield of the PDMS-bound BODIPY-H sample vs the solution sample. This may be due to better ISC in the nonsolvated BODIPY, originating from constrained rotation at the BODIPY-aryl linkage. ${ }^{30}$ Differences between $\tau_{1}$ and $\tau_{2}$ in the solution and solid phase are consistent with those observed for other dyes absorbed into PDMS (rather than covalently linked). ${ }^{10}$

As the desired bactericidal agent is ${ }^{1} \mathrm{O}_{2}$, these data were good preliminary indications that the modified PDMS would act as effective light-activated bactericidal surfaces. In particular, the data highlighted that BODIPY-Br would show increased lightactivated bactericidal activity compared to that of BODIPY- $\mathrm{H}$. Materials characterization show that the BODIPY was successfully surface-bound to the PDMS, with no detrimental effects from the covalent coupling on its photoactivity. A further advantage to this strategy is that a large amount of free material can be reclaimed from the surface functionalization process and thus recycled, improving efficiency. Finally, the predominance of the dye at the surface ensures that material is not wasted through trapping in the bulk.

The light-activated bactericidal potency of the PDMS-H and -Br samples was tested against the Gram-positive bacterium, $S$. aureus 8325-4. Samples were inoculated with the bacteria and then either irradiated with bright hospital white lighting (4000 $\mathrm{x}^{12}$ ) or maintained under dark conditions for up to $5 \mathrm{~h}$. The number of colony-forming units (CFU) was then determined.

In line with the ${ }^{1} \mathrm{O}_{2}$ generation measurements, the two BODIPY-modified PDMS samples showed markedly different light-activated bactericidal activities; the BODIPY-H modified sample showed limited light-activated antibacterial activity compared with the PDMS control. The PDMS-Br sample, however, showed potent light-activated bactericidal activity, achieving a reduction in bacterial numbers to below the detection limit within $3 \mathrm{~h}$ (Figures 4 and S7, kill is significant at the $p<0.001$ level by Student's $t$-test). That very little kill was observed with the PDMS-H, despite measurable production of ${ }^{1} \mathrm{O}_{2}$, may be justified in part by its weaker and slightly narrower absorption profile, reducing its effective absorption relative to 


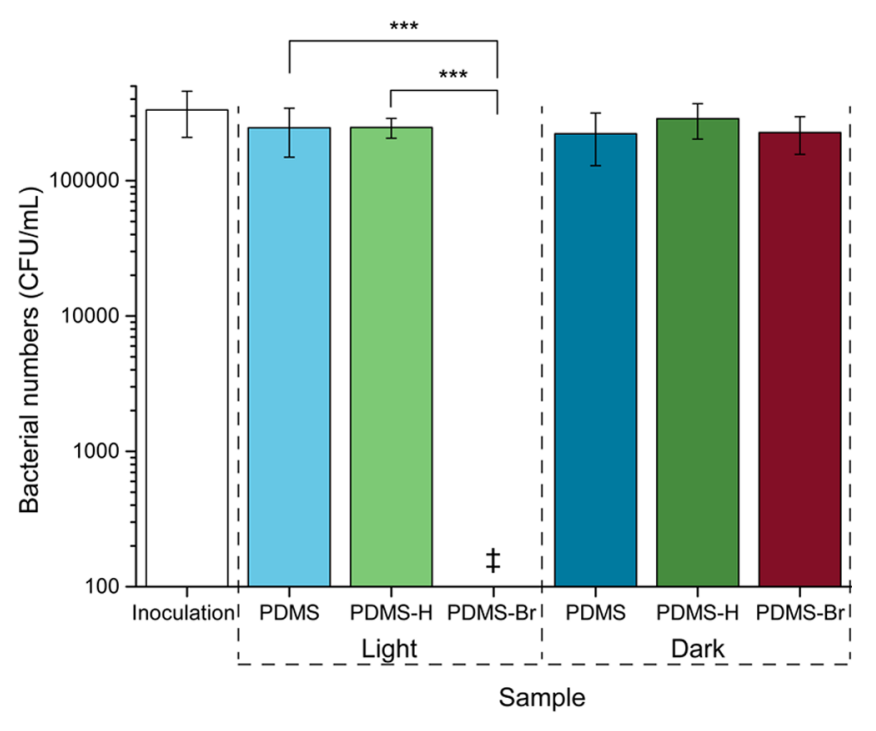

Figure 4. Antibacterial testing of PDMS-H and PDMS-Br in dark and light conditions against $S$. aureus. Illumination was $4000 \mathrm{~lx}$ of white hospital light for $5 \mathrm{~h}$. A $\$$ indicates at least $99.9 \%$ reduction in bacterial numbers to below the limit of detection of $100 \mathrm{CFU} / \mathrm{mL}$ and $* * *$ indicates significant difference at the $p<0.001$ level.

PDMS-Br. It should also be noted that other ROS (hydroxyl radicals, etc.) may be being formed but the presence of ${ }^{1} \mathrm{O}_{2}$ is unequivocally demonstrated by the previous phosphorescence decay measurements.

Further testing was carried out against Gram-negative Escherichia coli to investigate this more challenging target. Although $S$. aureus is a highly prevalent surface pathogen in hospital environments, ${ }^{31,32}$ it is more susceptible to the ROS generated by these materials than Gram-negative counterparts. It was found that $E$. coli could be reduced to below detectable levels within $20 \mathrm{~h}$ with PDMS-Br, suggesting that enough ROS are produced to damage Gram-negative species (Figure S8). At this time scale, an overall reduction in colony units was seen on the illuminated samples and further optimization would be required to claim high efficacy against E. coli.

\section{CONCLUSIONS}

We have demonstrated a covalently attached, photosensitized antimicrobial material, with potential applications in healthcare devices for preventing and treating hospital-acquired infections. Heavy-atom incorporation on the BODIPY sensitizer allowed for improved singlet oxygen generation, and antibacterial tests showed a highly significant reduction in the numbers of $S$. aureus from the Br-incorporated BODIPY, attached to PDMS, whereas in the absence of the $\mathrm{Br}$, little kill was observed, even after $5 \mathrm{~h}$.

It should be noted that it may be possible to synthesize an even more potent ${ }^{1} \mathrm{O}_{2}$-generating PDMS using an iodoBODIPY analogue. ${ }^{33-35}$ However, this was not pursued in this work as preliminary attempts resulted in low synthetic yields of the iodo-BODIPY and highlighted synthetic complications on subsequent covalent attachment steps. Although the BODIPY-Br sample synthesized achieved efficient bacterial kills, reducing viable counts to below the detection limit, the use of iodo-analogues might result in more rapid antimicrobial activity or prove effective under lower intensity lighting, widening applications to wards with low lighting conditions. Further to this, multiple BODIPYs might be attached to the polymer surface, thanks to the linking regime used. Therefore, utilization of quaternary bearing BODIPYs might assist in improving dark-kill. Finally, it may be beneficial to seek out a more hydrophilic dye or linker group to prevent surface rearrangement and encourage more dye to remain at the polymer/air interface. Future work will focus in this direction.

This paradigm of covalent attachment is facile and reduces waste of the sensitizer material in the bulk of the polymer matrix. The materials used are metal free, and therefore avoid issues of expense and associated health concerns from leaching. This technique also improves surface availability of the sensitizer, improving functional properties and paving the way toward a new generation of antimicrobial touch surfaces and devices.

\section{METHODS}

Reagents were used as supplied from Sigma-Aldrich or Alfa Aesar, without further purification. All water used was deionized $(>15 \mathrm{M} \Omega$ $\mathrm{cm})$ and reactions performed at room temperature (RT) $\left(\sim 20{ }^{\circ} \mathrm{C}\right)$ unless otherwise noted. All additional instrumental techniques and processes are detailed in the Supporting Information.

Materials Synthesis. BODIPY-H was prepared from a literature procedure. $^{36}$ In a $250 \mathrm{~mL}$ flask, 4-hydroxybenzaldehyde $(0.36 \mathrm{~g}, 3$ $\mathrm{mmol})$ and 2,4-dimethylpyrrole $(0.63 \mathrm{~g}, 6.6 \mathrm{mmol})$ were dissolved in THF $(90 \mathrm{~mL})$, with a few drops of triflouroacetic acid. This mixture was stirred at room temperature (RT) for $18 \mathrm{~h}$ before 2,3-dichloro-5,6dicyano-p-benzoquinone (DDQ) $(0.69 \mathrm{~g}, 3 \mathrm{mmol})$ in THF $(120 \mathrm{~mL})$ was added. The reaction was then stirred for another $4 \mathrm{~h}$ before cooling in an ice bath. Triethylamine $(18 \mathrm{~mL}, 0.12 \mathrm{~mol})$ was added dropwise, followed by $\mathrm{BF}_{3} \cdot \mathrm{OEt}_{2}(18 \mathrm{~mL}, 0.15 \mathrm{~mol})$, and green fluorescence was observed. The reaction was allowed to return to RT while stirring for $18 \mathrm{~h}$, then the crude product was filtered through a celite pad. The pad was washed with dichloromethane (DCM) (50 $\mathrm{mL}$ ) and the filtrate dried on a rotary evaporator. The crude solid was redissolved in DCM $(100 \mathrm{~mL})$ and washed with a saturated sodium bicarbonate solution $(50 \mathrm{~mL})$, then twice with water $(2 \times 50 \mathrm{~mL})$. The organic portion was dried over magnesium sulfate and purified with column chromatography (DCM) to give a red product (426.9 $\mathrm{mg}, 1.32 \mathrm{mmol}, 44 \%) .{ }^{1} \mathrm{H}$ NMR (500 MHz, CDCl 3 ): $\delta 7.14(2 \mathrm{H}, \mathrm{d}, J$ $=8.5 \mathrm{~Hz}), 6.95(2 \mathrm{H}, \mathrm{d}, J=8.5 \mathrm{~Hz}), 5.97(2 \mathrm{H}, \mathrm{s}), 4.91(1 \mathrm{H}, \mathrm{s}, \mathrm{OH})$, $2.55(6 \mathrm{H}, \mathrm{s}), 1.44(6 \mathrm{H}, \mathrm{s})$. HRMS $\mathrm{m} / z$ [EI+]: Theoretical 340.1673 , measured 340.1675. Elemental analysis $\mathrm{C}_{19} \mathrm{H}_{19} \mathrm{~N}_{2} \mathrm{OF}_{2}$ : Calcd: C, 67.08; H, 5.63; N, 8.24. Found: C, 66.70; H, 5.57; N, 8.46.

BODIPY-Br was prepared, using a modified literature procedure. ${ }^{37}$ BODIPY-H $(62 \mathrm{mg}, 0.19 \mathrm{mmol})$ was dissolved in $60 \mathrm{~mL}$ of DCM. Bromine $(28 \mu \mathrm{L} 0.54 \mathrm{mmol})$ in DCM $(7 \mathrm{~mL})$ was added dropwise over the course of an hour, and the mixture was left to stir for $2 \mathrm{~h}$ at RT. The resulting solution was washed with sodium thiosulfate (30 $\mathrm{mL}$ ), which was subsequently extracted with $30 \mathrm{~mL}$ of DCM. The organics were combined and dried over magnesium sulfate. The crude solution was concentrated and purified with column chromatography using DCM as the solvent to yield an orange/red solid (64 mg, 0.13 mmol, 69\%). ${ }^{1} \mathrm{H}$ NMR (500 MHz, $\left.\mathrm{CDCl}_{3}\right): \delta 7.11(2 \mathrm{H}, \mathrm{d}, J=8.4 \mathrm{~Hz})$, $6.99 \mathrm{ppm}(2 \mathrm{H}, \mathrm{d}, J=8.4 \mathrm{~Hz}), 4.96(1 \mathrm{H}, \mathrm{s}, \mathrm{OH}), 2.61(6 \mathrm{H}, \mathrm{s}), 1.44$ $(6 \mathrm{H}, \mathrm{s})$. HRMS $\mathrm{m} / \mathrm{z}$ [EI-]: Theoretical 493.9854, measured 493.9856. Elemental analysis $\mathrm{C}_{19} \mathrm{H}_{17} \mathrm{~N}_{2} \mathrm{OF}_{2} \mathrm{Br}_{2}$ : Calcd: C, 45.83; $\mathrm{H}$, 3.44; N, 5.63. Found: C, 45.53; H, 3.49; N, 5.64.

PDMS was prepared from a Dow Corning Sylgard 184 elastomer kit, as directed by the manufacturer. The elastomer ( $5 \mathrm{~g}$ in total) was premixed and then poured into $9 \mathrm{~cm}$ diameter Petri dishes before curing at RT for $72 \mathrm{~h}$. The cured polymer was then trimmed to ensure even thickness and cut into $0.5 \mathrm{~cm}$ wide strips for further treatment.

PDMS-S was prepared as described by Brook et al. ${ }^{25}$ using $28 \mathrm{~mL}$ of methanolic solution containing $\mathrm{w} / \mathrm{w} \quad 1 \% \mathrm{KOH}$ and $20 \%$ (3mercaptopropyl)trimethoxysilane (MTS). The PDMS was soaked in this solution for $8 \mathrm{~h}$ at $50{ }^{\circ} \mathrm{C}$. The polymer strips were rinsed with $\mathrm{MeOH}$ and sonicated in clean $\mathrm{MeOH}$ to remove surface-attached 
$\mathrm{KOH}$, followed by washing in DCM. The as-prepared polymer was stored under $\mathrm{MeOH}$ before use.

PDMS-MA was prepared by soaking the PDMS-S strips in $20 \mathrm{~mL}$ of THF solution, containing $4 \mathrm{~g}$ of maleic anhydride and a catalytic amount of AIBN, for $36 \mathrm{~h}$ at $66^{\circ} \mathrm{C}$. The polymer was then washed for $48 \mathrm{~h}$ in THF, with the solvent being refreshed every $12 \mathrm{~h}$. PDMS-X where $\mathrm{X}=\mathrm{H} / \mathrm{Br}$ was prepared by soaking the polymer strips in a 3 $\mathrm{mg} / \mathrm{mL}$ DCM solution of the respective BODIPY-X for $72 \mathrm{~h}$. The polymer was then washed in DCM to remove noncovalently attached material for $>96 \mathrm{~h}$, with the solvent being refreshed every $24 \mathrm{~h}$, until no color was observed. The original DCM BODIPY solutions were retained and dried down for reuse (retrieval efficiency $=97 \%$ ).

Antibacterial Testing. Antibacterial testing was performed using S. aureus $8325-4$ or E. coli ATCC 25922 as the test bacterium, cultured as described in the Supporting Information, on the following set of samples $(0.5 \mathrm{~cm} \times 0.5 \mathrm{~cm})$ : PDMS, PDMS-H, or PDMS-Br. A washed suspension of culture was diluted 10000 -fold to obtain $10^{5} \mathrm{CFU} / \mathrm{mL}$. Ten microliters of bacterial suspension was placed onto each of the samples as a droplet, and the inoculated samples were then placed in Petri dishes containing wet filter paper to maintain humidity (not covered with coverslips). One set of replicates was exposed to white light, whereas another set was kept in the dark. After light exposure for a determined period of time, the samples were placed into $400 \mu \mathrm{L}$ of phosphate-buffered saline (PBS) and mixed using a vortex mixer for 1 min to ensure that the bacteria were resuspended in PBS. The bacterial suspension was plated onto mannitol salt agar and incubated at $37^{\circ} \mathrm{C}$ for $24 \mathrm{~h}$. The colonies that grew on the plates were counted.

\section{ASSOCIATED CONTENT}

\section{S Supporting Information}

The Supporting Information is available free of charge on the ACS Publications website at DOI: 10.1021/acsami.7b13273.

Experimental procedures, images, and characterization data for the materials (PDF)

\section{AUTHOR INFORMATION}

\section{Corresponding Authors}

*E-mail: william.peveler@glasgow.ac.uk (W.J.P.).

*E-mail: i.p.parkin@ucl.ac.uk (I.P.P.).

ORCID ${ }^{\circ}$

William J. Peveler: 0000-0002-9829-2683

Gi Byoung Hwang: 0000-0003-0874-8390

Colin R. Crick: 0000-0001-9674-3973

Joshua B. Edel: 0000-0001-5870-8659

\section{Present Address}

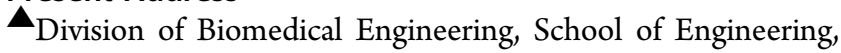
University of Glasgow, Glasgow G12 8LT, U.K.

\section{Notes}

The authors declare no competing financial interest.

\section{ACKNOWLEDGMENTS}

Donation of the fluorescence spectrometer was gratefully received from Richard H. Unthank. W.J.P. is recipient of an EPSRC Doctoral Prize Fellowship (EP/M506448/1). S.N. and C.R.C. acknowledge the Ramsay Trust for a Memorial Fellowship.

\section{REFERENCES}

(1) Weber, D. J.; Anderson, D.; Rutala, W. A. The Role of the Surface Environment in Healthcare-Associated Infections. Curr. Opin. Infect. Dis. 2013, 26, 338-344.

(2) Weinstein, R. A. Epidemiology and Control of Nosocomial Infections in Adult Intensive Care Units. Am. J. Med. 1991, 91, S179S184.
(3) Noimark, S.; Dunnill, C. W.; Parkin, I. P. Shining Light on Materials-a Self-Sterilising Revolution. Adv. Drug Delivery Rev. 2013, $65,570-580$.

(4) Hasan, J.; Crawford, R. J.; Ivanova, E. P. Antibacterial Surfaces: the Quest for a New Generation of Biomaterials. Trends Biotechnol. 2013, 31, 295-304.

(5) Muller, M. P.; MacDougall, C.; Lim, M. Antimicrobial Surfaces to Prevent Healthcare-Associated Infections: a Systematic Review. J. Hosp. Infect. 2016, 92, 7-13.

(6) Noimark, S.; Weiner, J.; Noor, N.; Allan, E.; Williams, C. K.; Shaffer, M. S. P.; Parkin, I. P. Dual-Mechanism Antimicrobial Polymer$\mathrm{ZnO}$ Nanoparticle and Crystal Violet-Encapsulated Silicone. Adv. Funct. Mater. 2015, 25, 1367-1373.

(7) Bovis, M. J.; Noimark, S.; Woodhams, J. H.; Kay, C. W. M.; Weiner, J.; Peveler, W. J.; Correia, A.; Wilson, M.; Allan, E.; Parkin, I. P. Photosensitisation Studies of Silicone Polymer Doped with Methylene Blue and Nanogold for Antimicrobial Applications. RSC Adv. 2015, 5, 54830-54842.

(8) Wainwright, M. Photoantimicrobials-So What's Stopping Us? Photodiagn. Photodyn. Ther. 2009, 6, 167-169.

(9) Durantini, A. M.; Greene, L. E.; Lincoln, R.; Martínez, S. R.; Cosa, G. Reactive Oxygen Species Mediated Activation of a Dormant Singlet Oxygen Photosensitizer: From Autocatalytic Singlet Oxygen Amplification to Chemicontrolled Photodynamic Therapy. J. Am. Chem. Soc. 2016, 138, 1215-1225.

(10) Noimark, S.; Salvadori, E.; Gómez-Bombarelli, R.; MacRobert, A. J.; Parkin, I. P.; Kay, C. W. M. Comparative Study of Singlet Oxygen Production by Photosensitiser Dyes Encapsulated in Silicone: Towards Rational Design of Anti-Microbial Surfaces. Phys. Chem. Chem. Phys. 2016, 18, 28101-28109.

(11) Noimark, S.; Dunnill, C. W.; Kay, C. W. M.; Perni, S.; Prokopovich, P.; Ismail, S.; Wilson, M.; Parkin, I. P. Incorporation of Methylene Blue and Nanogold Into Polyvinyl Chloride Catheters; a New Approach for Light-Activated Disinfection of Surfaces. J. Mater. Chem. 2012, 22, 15388-15396.

(12) Noimark, S.; Allan, E.; Parkin, I. P. Light-Activated Antimicrobial Surfaces with Enhanced Efficacy Induced by a DarkActivated Mechanism. Chem. Sci. 2014, 5, 2216-2223.

(13) Macdonald, T. J.; Wu, K.; Sehmi, S. K.; Noimark, S.; Peveler, W. J.; du Toit, H.; Voelcker, N. H.; Allan, E.; MacRobert, A. J.; Gavriilidis, A. Thiol-Capped Gold Nanoparticles Swell-Encapsulated Into Polyurethane as Powerful Antibacterial Surfaces Under Dark and Light Conditions. Sci Rep 2016, 6, No. 39272.

(14) Sehmi, S. K.; Noimark, S.; Bear, J. C.; Peveler, W. J.; Bovis, M.; Allan, E.; MacRobert, A. J.; Parkin, I. P. Lethal Photosensitisation of Staphylococcus aureus and Escherichia coli Using Crystal Violet and Zinc Oxide-Encapsulated Polyurethane. J. Mater. Chem. B 2015, 3, 64906500.

(15) Sehmi, S. K.; Noimark, S.; Weiner, J.; Allan, E.; MacRobert, A. J.; Parkin, I. P. Potent Antibacterial Activity of Copper Embedded Into Silicone and Polyurethane. ACS Appl. Mater. Interfaces 2015, 7, 22807-22813.

(16) Crick, C. R.; Noimark, S.; Peveler, W. J.; Bear, J. C.; Ivanov, A. P.; Edel, J. B.; Parkin, I. P. Advanced Analysis of Nanoparticle Composites - a Means Toward Increasing the Efficiency of Functional Materials. RSC Adv. 2015, 5, 53789-53795.

(17) Crick, C. R.; Noimark, S.; Peveler, W. J.; Bear, J. C.; Ivanov, A. P.; Edel, J. B.; Parkin, I. P. Advanced Compositional Analysis of Nanoparticle-Polymer Composites Using Direct Fluorescence Imaging. J. Visualized Exp. 2016, No. e54178.

(18) Felgenträger, A.; Maisch, T.; Späth, A.; Schröder, J. A.; Bäumler, W. Singlet Oxygen Generation in Porphyrin-Doped Polymeric Surface Coating Enables Antimicrobial Effects on Staphylococcus aureus. Phys. Chem. Chem. Phys. 2014, 16, 20598-20607.

(19) Ethirajan, M.; Chen, Y.; Joshi, P.; Pandey, R. K. The Role of Porphyrin Chemistry in Tumor Imaging and Photodynamic Therapy. Chem. Soc. Rev. 2011, 40, 340-362. 
(20) Kamkaew, A.; Lim, S. H.; Lee, H. B.; Kiew, L. V.; Chung, L. Y.; Burgess, K. BODIPY Dyes in Photodynamic Therapy. Chem. Soc. Rev. 2013, 42, 77-88.

(21) Ozdemir, T.; Bila, J. L.; Sozmen, F.; Yildirim, L. T.; Akkaya, E. U. Orthogonal Bodipy Trimers as Photosensitizers for Photodynamic Action. Org. Lett. 2016, 18, 4821-4823.

(22) Tekdaş, D. A.; Viswanathan, G.; Topal, S. Z.; Looi, C. Y.; Wong, W. F.; Tan, G. M. Y.; Zorlu, Y.; Gürek, A. G.; Lee, H. B.; Dumoulin, F. Antimicrobial Activity of a Quaternized BODIPY Against Staphylococcus Strains. Org. Biomol. Chem. 2016, 14, 2665-2670.

(23) Carpenter, B. L.; Scholle, F.; Sadeghifar, H.; Francis, A. J.; Boltersdorf, J.; Weare, W. W.; Argyropoulos, D. S.; Maggard, P. A.; Ghiladi, R. A. Synthesis, Characterization, and Antimicrobial Efficacy of Photomicrobicidal Cellulose Paper. Biomacromolecules 2015, 16, 2482-2492.

(24) Jóźwiak, A. B.; Kielty, C. M.; Black, R. A. Surface Functionalization of Polyurethane for the Immobilization of Bioactive Moieties on Tissue Scaffolds. J. Mater. Chem. 2008, 18, 2240-2248.

(25) Zhang, J.; Chen, Y.; Brook, M. A. Facile Functionalization of PDMS Elastomer Surfaces Using Thiol-Ene Click Chemistry. Langmuir 2013, 29, 12432-12442.

(26) Yang, S.; Khare, K.; Lin, P. C. Harnessing Surface Wrinkle Patterns in Soft Matter. Adv. Funct. Mater. 2010, 20, 2550-2564.

(27) Tanielian, C.; Golder, L.; Wolff, C. Production and Quenching of Singlet Oxygen by the Sensitizer in Dye-Sensitized PhotoOxygenations. J. Photochem. 1984, 25, 117-125.

(28) Gorman, A.; Killoran, J.; O’Shea, C.; Kenna, T.; Gallagher, W. M.; O'Shea, D. F. In Vitro Demonstration of the Heavy-Atom Effect for Photodynamic Therapy. J. Am. Chem. Soc. 2004, 126, 1061910631.

(29) Frimannsson, D. O.; Grossi, M.; Murtagh, J.; Paradisi, F.; O'Shea, D. F. Light Induced Antimicrobial Properties of a Brominated Boron Difluoride (BF 2) Chelated Tetraarylazadipyrromethene Photosensitizer. J. Med. Chem. 2010, 53, 7337-7343.

(30) Zhang, X.-F.; Yang, X. Singlet Oxygen Generation and Triplet Excited-State Spectra of Brominated BODIPY. J. Phys. Chem. B 2013, 117, 5533-5539.

(31) Dancer, S. J. Importance of the Environment in MeticillinResistant Staphylococcus aureus Acquisition: the Case for Hospital Cleaning. Lancet Infect. Dis. 2008, 8, 101-113.

(32) Joseph Omololu, A. S. Staphylococcus aureus Surface Colonization of Medical Equipment and Environment, Implication in Hospital- Community Epidemiology. J. Hosp. Med. Manage. 2017, 3, 3.

(33) Carpenter, B. L.; Situ, X.; Scholle, F.; Bartelmess, J.; Weare, W.; Ghiladi, R. Antiviral, Antifungal and Antibacterial Activities of a BODIPY-Based Photosensitizer. Molecules 2015, 20, 10604-10621.

(34) Orlandi, V. T.; Rybtke, M.; Caruso, E.; Banfi, S.; Tolker-Nielsen, T.; Barbieri, P. Antimicrobial and Anti-Biofilm Effect of a Novel BODIPY Photosensitizer Against Pseudomonas aeruginosa PAO1. Biofouling 2014, 30, 883-891.

(35) Caruso, E.; Banfi, S.; Barbieri, P.; Leva, B.; Orlandi, V. T. Synthesis and Antibacterial Activity of Novel Cationic BODIPY Photosensitizers. J. Photochem. Photobiol., B 2012, 114, 44-51.

(36) Liu, J.-Y.; Yeung, H.-S.; Xu, W.; Li, X.; Ng, D. K. P. Highly Efficient Energy Transfer in Subphthalocyanine-BODIPY Conjugates. Org. Lett. 2008, 10, 5421-5424.

(37) Jiao, L.; Pang, W.; Zhou, J.; Wei, Y.; Mu, X.; Bai, G.; Hao, E. Regioselective Stepwise Bromination of Boron Dipyrromethene (BODIPY) Dyes. J. Org. Chem. 2011, 76, 9988-9996. 